\title{
Density functional study of gallium clusters on graphene: electronic doping and diffusion
}

\author{
D. Nezval ${ }^{1,2}$, M. Bartošík ${ }^{1,2,3}$, J. Mach ${ }^{1,2}$, J. Piastek ${ }^{1,2}$, V. Švarc $^{1,2}$, M. Konečný $\dot{y}^{1,2}$ and T. Šikola ${ }^{1,2}$ \\ ${ }^{1}$ Institute of Physical Engineering, Brno University of Technology, Technická 2, 61669 Brno, Czech \\ Republic
}

${ }^{2}$ Central European Institute of Technology, Brno University of Technology, Purkyňova 656/123, 612 00 Brno, Czech Republic

${ }^{3}$ Department of Physics and Materials Engineering, Faculty of Technology, Tomas Bata University in Zlín, Vavrečkova 275, 76001 Zlín, Czech Republic

E-mail: mira.bartosik@gmail.com

\begin{abstract}
Motivated by experimental results on transport properties of graphene covered by gallium atoms, the density functional theory study of clustering of gallium atoms on graphene (up to a size of 8 atoms) is presented. The paper explains a rapid initial increase of graphene electron doping by individual $\mathrm{Ga}$ atoms with Ga coverage, which is continually reduced to zero, when bigger multiple-atom clusters have been formed. According to DFT calculations with and without the van der Waals correction, gallium atoms start to form a three-dimensional cluster from five and three atoms, respectively. The results also explain an easy diffusion of Ga atoms while forming clusters caused by a small diffusion barrier of $0.11 \mathrm{eV}$. Moreover, the calculations show this barrier can be additionally reduced by the application of an external electric field, which was simulated by the ionization of graphene. This effect offers a unique possibility to control the cluster size in experiments only by applying a gate-voltage to the graphene in a field-effect transistor (FET) geometry and thereby without growth temperature assistance.
\end{abstract}

Keywords: graphene, gallium, DFT, clusters, doping, surface diffusion

\section{Introduction}

Graphene is a single two-dimensional layer of carbon atoms arranged in a hexagonal structure. It is one of the most studied materials since its experimental verification [1,2]. This is because of graphene's excellent electrical [3-6], mechanical $[7,8]$ and optical $[9,10]$ properties. These properties result from 
$\mathrm{sp}^{2}$ bonds between carbon atoms and unique electronic structure with the conical dispersion at $\mathrm{K}$ points of the Brillouin zone, where the conduction and valence bands touch each other. In order to use graphene in real devices, it is necessary to consider the influence of ambient atoms, which can change the pristine graphene electronic structure and cause the band gap opening or a shift of the Fermi level resulting in electronic doping of graphene [11-13].

Metallic elements reveal a diversity of properties, and when used properly, they can modify and control graphene electronic or magnetic properties. It was studied both experimentally $[13,14]$ and theoretically [12,15-17]. Especially, a gold-graphene interface has been frequently investigated because of its widespread application as metal electrodes $[12,13,15,18]$. It was proved experimentally that the adsorption of gold, antimony and bismuth lead to hole doping of graphene [13]. A useful tool for understanding how adsorbed atoms affect graphene electronic properties is the density functional theory (DFT). Chan et al. [19] and Ishii et al. [20] carried out DFT calculations of single-metal-adatom adsorption on graphene. Cao et al. [21] and Johll et al. [22] performed the first-principles studies of transition metal adatoms and dimers on graphene. DFT calculations of gold clusters on graphene were published by Srivastava et al. [12] and Amft et al. [15] Several more first-principles studies were focused on metal clusters adsorption on graphene (Pd [23-25], Zr [26], Fe [12], Ni [24,27], Pt [17,24,25], Ag $[28,29])$.

The DFT gives good results in the case of structures possessing strong bonds. Unfortunately, this method becomes less precise when the interaction between atoms is weak and so the long-range van der Waals (vdW) forces should be taken into account. This type of interactions are not implemented in default DFT calculations and so in the case of need it has to be implemented in the form of corrections. It has been found, that DFT with local and semi-local exchange correlations gives low binding forces between graphene and noble metal adatoms $[30,31]$ and, hence, a vdW correction is needed. Similarly, the observations of Srivastava et al. [12] show Au clusters are weakly bonded to graphene and thus long-range vdW dispersion forces must be taken into account in the calculations as well. One of the most used types of vdW corrections is a semiempirical method DFT-D2 by Grimme [12,32,33]. 
Gallium is a promising element for the graphene based electronic devices. It is a component of GaAs/graphene solar cells [34,35], Ga/graphene systems applied in ultraviolet plasmonics [36,37], and it is a good template for heteroepitaxial growth of GaN [38]. Several experimental studies [37,39] focused on the deposition of atomic Ga on graphene proved cluster growth of Ga, but almost no DFT computational study (see our previous work ${ }^{34}$ ) has been performed for Ga-atom cluster forming on graphene. The attention was paid only to a substitution of carbon atoms in graphene grid by $\mathrm{Ga}$ and $\mathrm{Ge}$ atoms which strongly transforms graphene band structure itself [40].

In this work the adsorption of Ga clusters containing up to 8 atoms on graphene is investigated. These first principle calculations explain the changes of graphene electronic properties with the size of Ga clusters, especially, the influence on graphene doping and its saturation [39]. The incorporation of a van der Waals interaction correction justified by small binding energy between Ga atoms and graphene has made the calculation more realistic. Furthermore, the calculation of diffusion (barriers) of Ga on intrinsic and doped graphene was performed. It gives an insight for experimentalist how the cluster formation can be influenced by the temperature of graphene and its electrical doping by e. g. gate voltage in a graphene field-effect-transistor (FET) arrangement [37,39].

\section{Methods}

The DFT calculations were performed using the projected augmented wave (PAW) $[41,42]$ method implemented in the Vienna Ab-initio Simulation Package (VASP) [43-45]. The generalized gradient approximation (GGA) proposed by Perdew, Burke and Ernzerrhorf (PBE) [46] was adapted to treat the electronic exchange and correlation, and all calculations were recalculated with the vdW correction DFT-D2 method by Grimme [32]. All calculations were performed with an $8 \times 8$ graphene supercell containing 128 carbon atoms and the desired amount of Ga atoms. A $17 \AA$ vacuum spacer was used to eliminate the interaction between layers in the laterally neighboring supercells. The energy cut-off was set up to a value of $600 \mathrm{eV}$. A $\Gamma$-centered 3x3x1 Monkhorst-Pack mesh [47] was used for structure optimizations. For the calculation of the density of states (DOS) a 9x9x1 mesh was used. All structures were relaxed with a tolerance of the residual forces of $0.01 \mathrm{eV} \AA^{-1}$. 

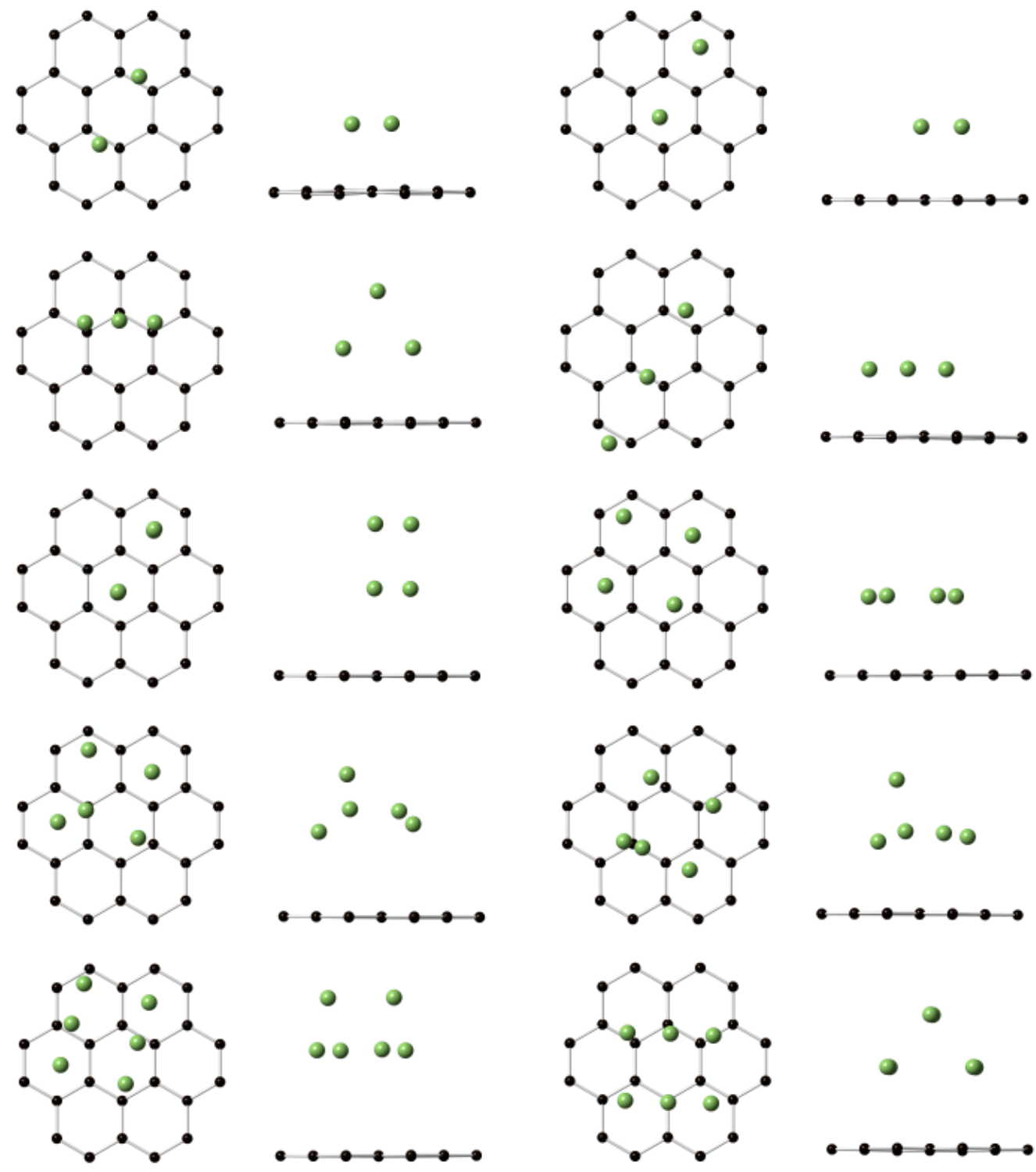

00
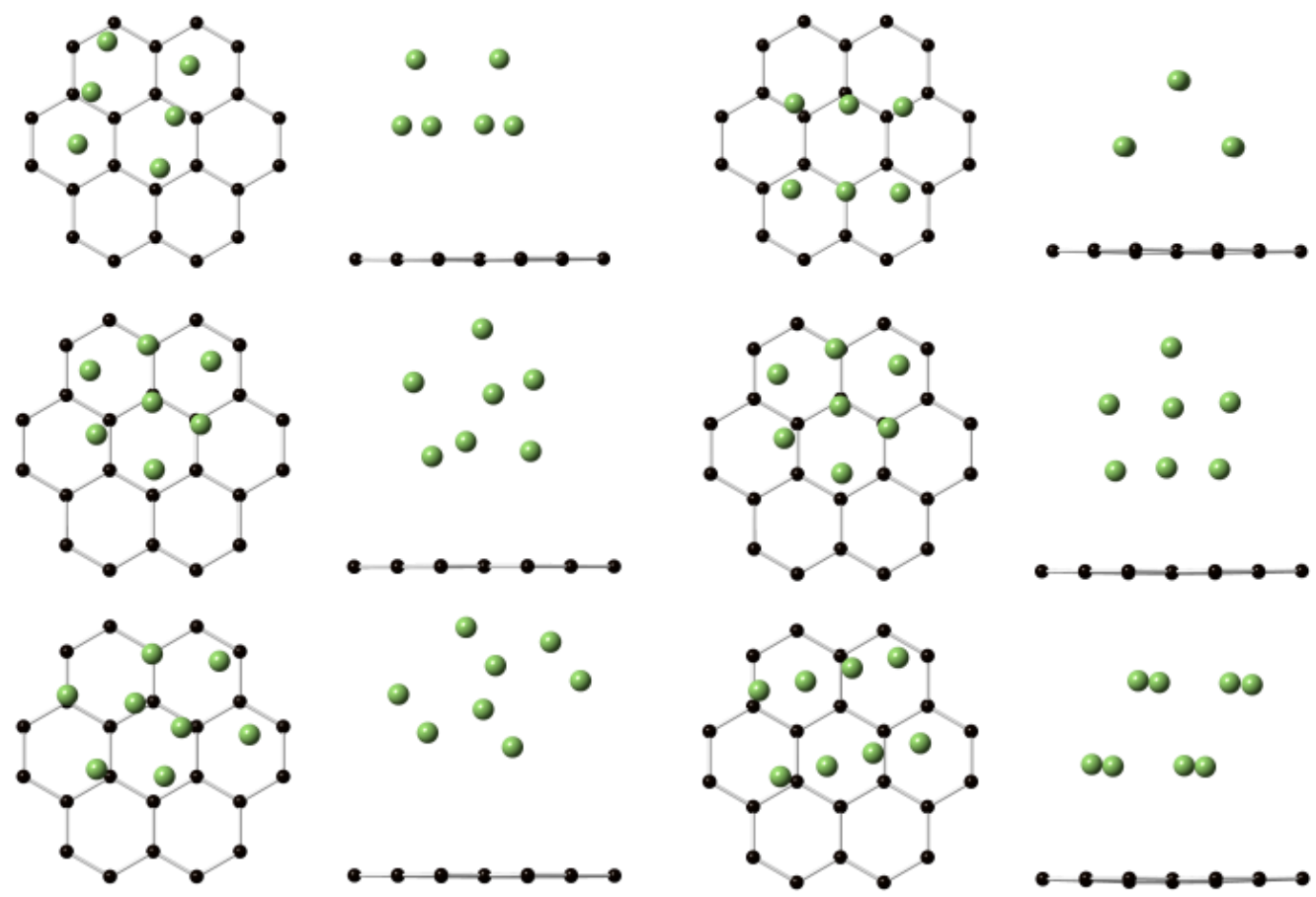

๑๐ ค०

○० 00

FIG. 1.: Optimized geometry of Ga clusters adsorbed on graphene from top and side views. Clusters were studied for the size from 2 to 8 atoms. PBE calculations are on the left and vdW corrected calculations are on the right. 


\section{Results}

The low binding energy of Ga clusters to graphene led us to take into account the long-range vdW forces. All structures with Ga clusters were calculated without corrections (PBE) and with corrections (DFT-D2). The clusters of gallium atoms on graphene were examined up to a size of 8 atoms in the cluster. The final arrangements of atoms in clusters (Fig. 1) have the lowest total energy. To find the minimal energy, the different initial conformations of atoms were relaxed. These conformations were based on the geometry of one-atom smaller cluster by adding an atom in several reasonable positions and on typical clump shapes published in literature $[19,48]$. In the case of calculations without vdW corrections, the Ga atoms start creating 3D clusters from a size of 3 atoms (Fig. 1 left). In contrast, the $\mathrm{vdW}$ correction results in higher adhesion and clusters up to a size of 4 atoms remain in the planar arrangement parallel to the graphene sheet (Fig. 1 right).

TABLE I.: Number of atoms in the cluster $\left(N_{\mathrm{Ga}}\right)$, the shift of the Fermi level above the Dirac point $\left(\Delta E_{F}\right)$, charge transfer $(\Delta q)$, the height of the nearest Ga atom above graphene $(h)$ and binding energy $\left(E_{b}\right)$ of Ga clusters adsorbed on graphene. All results are without and with vdW corrections.

\begin{tabular}{|c|c|c|c|c|c|c|c|c|}
\hline \multirow{2}{*}{$N_{\mathrm{Ga}}$} & \multicolumn{2}{|c|}{$\Delta E_{F}(\mathrm{eV})$} & \multicolumn{2}{c|}{$\Delta q(\mathrm{e})$} & \multicolumn{2}{c|}{$h(\AA)$} & \multicolumn{2}{c|}{$E_{b}(\mathrm{eV})$} \\
\cline { 2 - 9 } & PBE & DFT-D2 & PBE & DFT-D2 & PBE & DFT-D2 & PBE & DFT-D2 \\
\hline 1 & 0.51 & 0.51 & 0.62 & 0.63 & 2.21 & 2.19 & 0.987 & 1.09 \\
\hline 2 & 0.58 & 0.58 & 0.60 & 0.57 & 2.70 & 2.75 & 0.645 & 0.780 \\
\hline 3 & 0.17 & 0.51 & 0.31 & 0.84 & 2.85 & 2.60 & 0.334 & 0.582 \\
\hline 4 & 0 & 0.46 & 0.13 & 0.38 & 3.28 & 2.95 & 0.033 & 0.321 \\
\hline 5 & 0 & 0.28 & 0.24 & 0.40 & 3.22 & 2.73 & 0.041 & 0.334 \\
\hline 6 & 0 & 0 & 0.042 & 0.24 & 3.54 & 3.05 & 0.022 & 0.158 \\
\hline 7 & 0 & 0 & 0.22 & 0.39 & 3.14 & 2.82 & 0.031 & 0.210 \\
\hline 8 & 0 & 0 & 0.035 & 0.064 & 3.62 & 3.13 & 0.011 & 0.091 \\
\hline
\end{tabular}


(a)



(c)

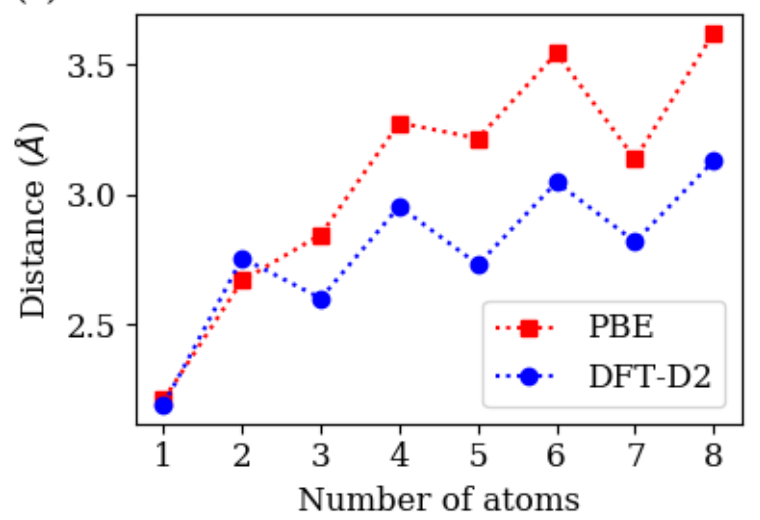

(b)

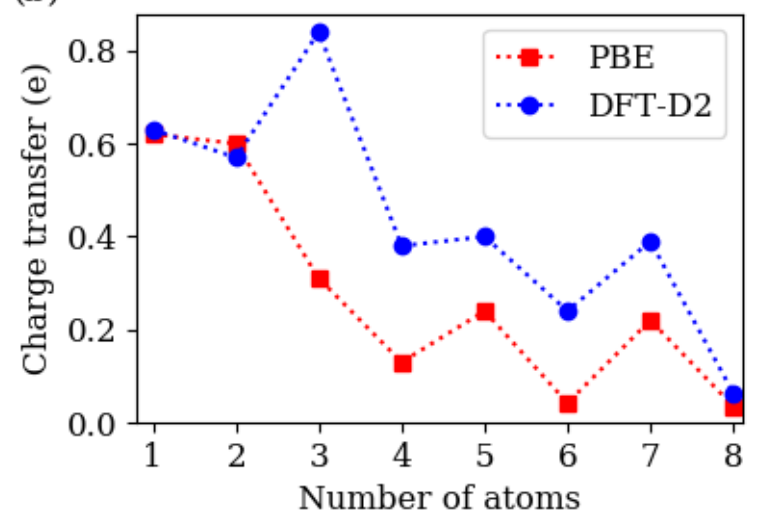

(d)

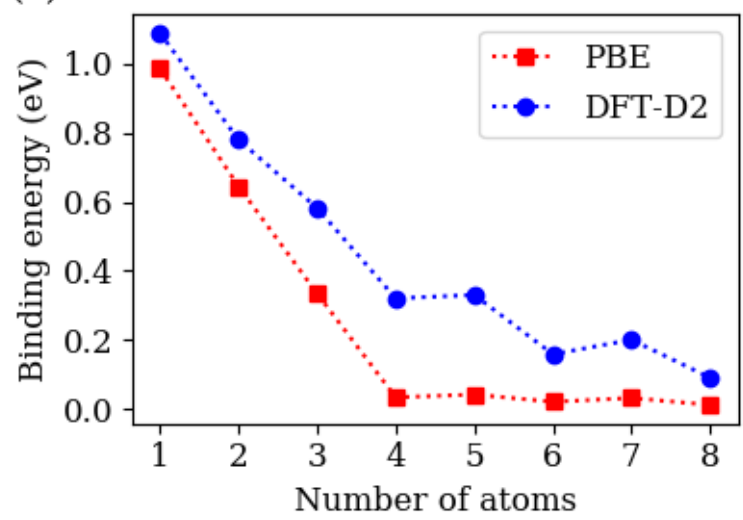

FIG. 2.: (a) The Fermi level shift above the Dirac point of graphene, (b) the charge transfer of electrons from $\mathrm{Ga}$ into the graphene, (c) the distance of the closest $\mathrm{Ga}$ atom from graphene, and (d) the binding energy of Ga clusters on graphene as a function of $\mathrm{Ga}$ atom number in clusters.

The next steps were aimed at the calculation of the band structure, density of states and Bader charge analysis [49]. The data are graphically shown in Fig. 2 (a-d) and the values in Table I. The electron doping of graphene decreases with the size of the cluster (Fig. 2a). The band structure results show that Ga clusters stop changing the Fermi level (Fig. 2a) and so doping of graphene from a size of 4 and 6 atoms for calculation with and without the vdW correction, respectively. In the case of calculations with the correction, the electron doping effect is higher. The Bader charge analysis (Fig. 2b) leads to interesting oscillations for odd and even number of atoms in the cluster, but the overall trend agrees with the Fermi level shift. With the size of clusters, the transport of electrons to graphene decreases and again for calculations with the vdW correction the transfer of electron is higher. Generally, the distance between the graphene layer and the closest Ga atom increases with the number of atoms in cluster (Fig. 2c). This is in logical agreement with the decrease of binding energy of Ga clusters to 
graphene as a function of cluster size (Fig. 2d). We can see that the vdW correction influences the absolute values of respective quantities, but the trends are similar for both approaches. The oscillations for odd and even number of atoms in the cluster is also present in the results of the distance between the graphene layer and the closest Ga atom (Fig. 2c) and, less profoundly in the results of the binding energy of the whole cluster to graphene (Fig. 2d). The results show that in the case of odd numbers of atoms, which corresponds to odd numbers of electrons, the individual atom interacts more intensely with graphene, which leads to a bigger charge transfer and binding energy and to a smaller distance between Ga atoms and graphene. Srivastava et al. [12] published similar behavior - the odd number of Au atoms in cluster doped graphene and the even number did not dope at all.

The main decreasing trends in doping of graphene (Fig. 2 a,b), binding energy of Ga cluster to graphene (Fig. 2d), and the increasing trend of gallium cluster - graphene distance (Fig. 2c) with the number of atoms in cluster can be explained as follows. The gallium preferentially uses its valence electrons to form bonds with other Ga atoms, and only then to form bonds with graphene and its doping. Therefore, the bigger Ga cluster consumes on average more electrons to bind $\mathrm{Ga}$ atoms together and fewer electrons are left to form bonds with graphene and its doping.

The subtle oscillations (Fig. 2 b-d) are related to a gallium electron configuration, in which there is one unpaired electron in the last shell $\left(4 \mathrm{p}^{1}\right)$. Consequently, in the case of odd-atom gallium cluster the remaining Ga odd-electron is used for binding and doping of graphene. On the other hand, in the case of even-atom cluster the electron pairs are preferentially involved in binding of gallium atom pairs together, and the transfer of electrons into the graphene is partially reduced. In other words, the evenatom clusters contain mainly compensated electron pairs binding gallium atom pairs in cluster together, however, odd-atom clusters also contain unpaired electrons interacting with graphene. 

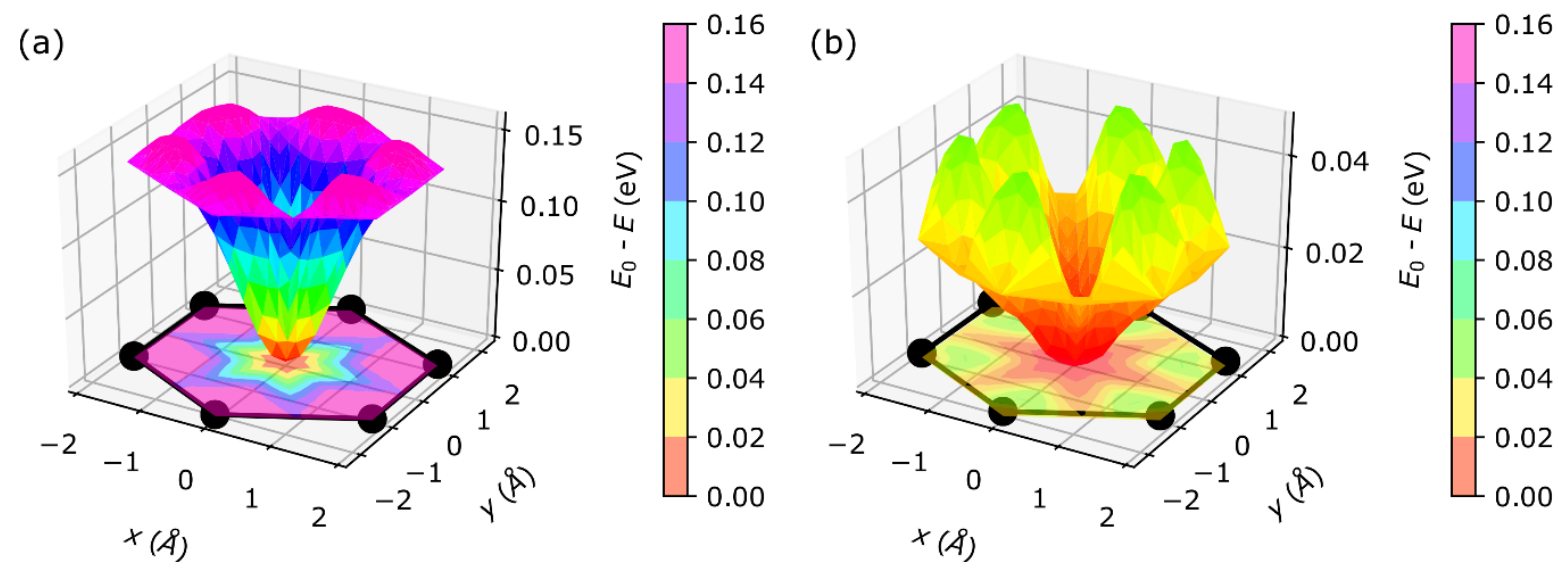

(c)

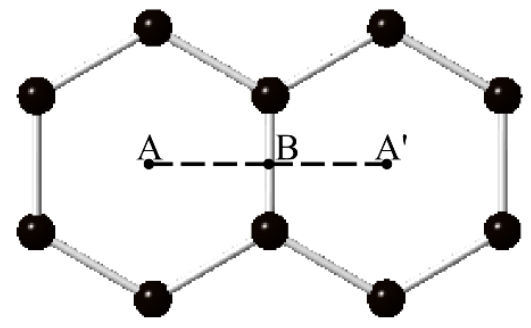

(d)



FIG. 3.: Total energy difference as a function of $\mathrm{Ga}$ atom position above graphene hexagon for (a) intrinsic graphene and (b) ionized graphene (n-doped by adding 3 electrons to the calculated system). (c) The optimal path for Ga diffusion on the graphene sheet. (d) The energy diffusion barrier along the optimal path for intrinsic and ionized graphene.

The creation of clusters is significantly influenced by the diffusion barrier for Ga atoms motion over the graphene sheet. The calculation results of the energy diffusion barrier for an individual Ga atom on graphene are presented in Fig. 3. First, the total energy of the $\mathrm{Ga}$-graphene system in the dependency on a Ga atom position on a graphene hexagon was calculated. The Ga atom was fixed in $x$ and $y$ position and a relaxed with respect to the $\mathrm{z}$ coordinate for each $x, y$ position. The energies shown in Fig. 1 (a) are the differences between the total energies of the system with the Ga atom in a given position and with the Ga atom above the center of the graphene hexagon corresponding to the energy minimum. The calculations were carried out without the vdW correction since the individual atom is adsorbed with higher binding energy. The diffusion path between two equivalent central positions is less energy demanding in the case of crossing over the C-C bond as it is shown in Fig. 3(c). The energy diffusion barrier that must be overcome is $0.14 \mathrm{eV}$ (Fig. 3(d)). This is a small value compared to barriers of 0.75 $\mathrm{eV}$ and $0.5 \mathrm{eV}$ for a $\mathrm{Zr}$ adatom [24] and $\mathrm{C}$ adatom [50] on graphene, respectively. The diffusion barrier 
was even lower $0.11 \mathrm{eV}$ when the vdW correction was incorporated. This means, the individual Ga atoms can easily diffuse along with the graphene sheet.

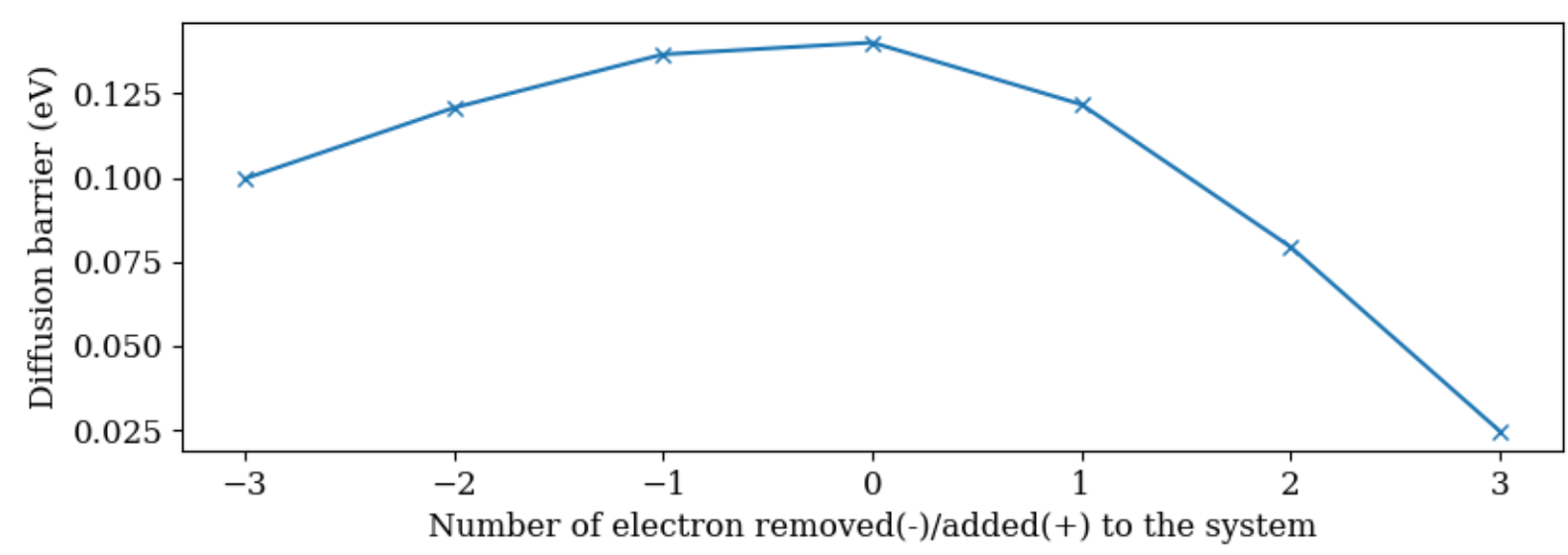

FIG. 4.: Diffusion energy barrier as a function of the ionization degree of the $8 \times 8$ graphene supercell and one $\mathrm{Ga}$ atom. The negative values on the $x$ axis correspond to removing electrons from the system which cause positive charging, the positive values to adding electrons to the system and negative charging.

In the next step, the changes in Ga diffusion caused by the charging of graphene was investigated. It was realized by adding or removing electrons in the system consisting of the graphene sheet and one $\mathrm{Ga}$ atom. The maximum number of added or removed electrons per an $8 \times 8$ graphene supercell was 3, which is equal to a charge concentration of $8.89 \cdot 10^{13} \mathrm{e}^{-/} / \mathrm{cm}^{2}$. Generally, the doping of graphene by charge carriers decrease the diffusion barrier, no matter which doping polarity was chosen, as shown in Fig. 4. If the electrons are removed from the system (p-doping), the diffusion barrier is decreased to $0.10 \mathrm{eV}$ (from an original value of $0.14 \mathrm{eV}$ ) for the highest calculated doping. Even more significant decrease occurred in the case of n-doping, where the diffusion barrier for the highest doping was only $0.025 \mathrm{eV}$. This large reduction of a diffusion barrier caused by a presence of 3 additional electrons in the system can be also clearly seen in Fig. 3. (b) and (d). It should significantly enhance the diffusion of Ga atoms along a negatively charged graphene sheet.

Moreover, the asymmetry between the adding and removing electrons is visible in Fig. 4. The reason could be in initial n-doping of intrinsic graphene given by $\mathrm{Ga}$ atom itself. Consequently, the 
removing of electron from the system (p-doping) has lower effect on the reduction of barrier of graphene for a diffusing $\mathrm{Ga}$ atoms.

\section{Discussion}

The presented computational results are useful for the explanation of experimental results, and consequently for possible applications and limitations of Ga/graphene systems.

First, the Ga cluster size and its impact on the Fermi level position of graphene determines the amount of possible n-graphene doping by $\mathrm{Ga}$, which is limited to a certain saturation level. According to the calculation, the individual Ga atom brings on average 0.6 electrons into the graphene (Fig. $2 b$ ), however, the doping decreases with the Ga cluster size, and becomes negligible in the case of 6- and more-atom clusters (Fig. 2a). The fundamental reason for that is in the stronger binding of electrons between $\mathrm{Ga}$ atoms in the cluster, and consequent lower sharing of electrons with neighboring graphene substrate. It is also accompanied by an increasing distance between the Ga clusters and the graphene. It means, the higher Ga deposition above a certain level will not lead to higher doping. Hence, the doping effect is associated only with the clusters composed of less than 6 atoms. The calculations were performed for the maximum cluster size of 8 atoms at which the charge transfer from Ga into the graphene also disappears (Fig. 2b) and for larger clusters is negligible. The improved calculations bring corrections to our previous results [39] achieved without implementing the vdW interactions where only 3-atom clusters were considered. Such a Ga doping behavior is similar for other metals. For example, Srivasta $e t$ al. predicted the decrease of doping with a higher number of atoms in clusters for gold, and iron. They predicted the p-doping of graphene by gold in the range $0.12-0.02 \mathrm{e} / \mathrm{atom}$, and $\mathrm{n}$-doping by iron in the range $0.84-0.09$ for clusters composed of 1 to 5 atoms.

Second, the computation of Ga diffusion proves quite high mobility of Ga atoms on the graphene surface leading to a formation of clusters even at low concentrations of deposited Ga atoms at room temperature. In the case of carbon [50] and zirconium [26] on graphene, the diffusion barriers are five, respectively seven times higher than for Ga on graphene. Since most of the experiments dealing 
with Ga deposition on graphene are performed at room temperature, there is missing information on the influence of temperature on cluster formation. However, the diffusion barriers presented here will be of the same value, the thermal energy will enhance the cluster formation even at lower Ga deposited concentration.

Third, the calculation of the influence of the Ga diffusion barrier on the graphene charge state indicates an alternative way of controlling the Ga cluster size to the classical thermal one. It is especially suitable for graphene exhibiting a clear ambipolar effect [51] since such doping can be achieved by gatevoltage in common FET graphene configurations. The negative doping achieved by an external voltage can then lead to a significant lowering of the diffusion barrier and the formation of bigger Ga clusters. In our case the doping by one electron per calculated supercell corresponds to a gate voltage of $1250 \mathrm{~V}$ applied to graphene placed on a $280 \mathrm{~nm}$-thick $\mathrm{SiO}_{2}$ substrate, which is a quite high value for application in experiments due to a critical value of $\mathrm{SiO}_{2}$ breakdown voltage. Smaller gate voltages up to $100 \mathrm{~V}$ that can be safely used would cause a reduction of the barrier in order of percentage units. However, in experiment it is possible to use an insulating substrate with different permittivity or thickness, so that the electron diffusion from gallium into the graphene sheet can be controlled by back-gate voltage more efficiently.

\section{Conclusion}

We have performed the calculation of gallium clusters on free-standing graphene up to a size of 8 atoms. Gallium creates 3D clusters from 3 or 5 atoms without and with the implemented vdW correction, respectively. Gallium atoms negatively dope the graphene. The doping value decreases with the size of clusters and it is negligible from the size of 6 atoms in cluster up. The vdW correction increases the absolute values of doping, but such a doping behavior with the increasing cluster size remains the same.

We have also investigated the diffusion of one gallium atom on the graphene sheet. Graphene creates a small diffusion barrier of $0.11 \mathrm{eV}$ to a $\mathrm{Ga}$ atom. This barrier can be additionally reduced by ionization (doping) of structures, which can be experimentally achieved by the application of a proper gate voltage 
to graphene assembled in the field-effect transistor configuration. Due to this diffusion barrier reduction, the formation of bigger Ga clusters at the same temperature can be achieved.

The presented theoretical work has two important experimental and practical impacts dealing with $\mathrm{Ga} /$ graphene system formation. First, the $\mathrm{n}$-doping of graphene by $\mathrm{Ga}$ is limited to a saturation level due to the formation of large no-doping clusters at higher Ga coverages. Second, the diffusion of Ga on graphene can be enhanced by an external electric field.

\section{Acknowledgements}

We acknowledge the support by the Grant Agency of the Czech Republic (grant No. 17-21413S). This work was also supported by The Ministry of Education, Youth and Sports from the Large Infrastructures for Research, Experimental Development and Innovations project "IT4Innovations National Supercomputing Center - LM2015070".

\section{References}

[1] Novoselov K S, Geim a K, Morozov S V, Jiang D, Katsnelson M I, Grigorieva I V, Dubonos $\mathrm{S} \mathrm{V}$ and Firsov a a 2005 Two-dimensional gas of massless Dirac fermions in graphene. Nature 438 197-200

[2] Novoselov K S, Jiang D, Schedin F, Booth T J, Khotkevich V V., Morozov S V. and Geim A K 2005 Two-dimensional atomic crystals Proceedings of the National Academy of Sciences of the United States of America 102 10451-3

[3] Du X, Skachko I, Barker A and Andrei E Y 2008 Approaching ballistic transport in suspended graphene. Nature nanotechnology 3 491-5

[4] Bolotin K I, Sikes K J, Jiang Z, Klima M, Fudenberg G, Hone J, Kim P and Stormer H L 2008 Ultrahigh electron mobility in suspended graphene Solid State Communications 146 351-5

[5] Castro Neto a. H, Peres N M R, Novoselov K S and Geim a. K 2009 The electronic properties of graphene Reviews of Modern Physics $\mathbf{8 1} 109-62$

[6] Goerbig M O 2011 Electronic properties of graphene in a strong magnetic field Reviews of Modern Physics $\mathbf{8 3}$

[7] Frank I W, Tanenbaum D M, van der Zande a. M and McEuen P L 2007 Mechanical properties of suspended graphene sheets Journal of Vacuum Science \& Technology B: Microelectronics and Nanometer Structures 252558

[8] Lee C, Wei X, Kysar J W and Hone J 2008 Measurement of the elastic properties and intrinsic strength of monolayer graphene. Science (New York, N.Y.) 321 385-8 
[9] Bonaccorso F, Sun Z, Hasan T and Ferrari a. C 2010 Graphene photonics and optoelectronics Nature Photonics 4 611-22

[10] Tredicucci A and Vitiello M S 2014 Device concepts for graphene-based terahertz photonics IEEE Journal on Selected Topics in Quantum Electronics 20 130-8

[11] Lee B, Chen Y, Duerr F, Mastrogiovanni D, Garfunkel E, Andrei E Y and Podzorov V 2010 Modification of electronic properties of graphene with self-assembled monolayers Nano Letters $102427-32$

[12] Srivastava M K, Wang Y, Kemper A F and Cheng H P 2012 Density functional study of gold and iron clusters on perfect and defected graphene Physical Review B - Condensed Matter and Materials Physics 85 1-15

[13] Gierz I, Riedl C, Starke U, Ast C R and Kern K 2008 Atomic hole doping of graphene Nano Letters 8 4603-7

[14] Pi K, McCreary K M, Bao W, Han W, Chiang Y F, Li Y, Tsai S W, Lau C N and Kawakami R K 2009 Electronic doping and scattering by transition metals on graphene Physical Review B Condensed Matter and Materials Physics 80 1-5

[15] Amft M, Sanyal B, Eriksson O and Skorodumova N V. 2011 Small gold clusters on graphene, their mobility and clustering: A DFT study Journal of Physics Condensed Matter 23 1-10

[16] Song C, Ge Q and Wang L 2005 DFT studies of Pt/Au bimetallic clusters and their interactions with the CO molecule Journal of Physical Chemistry B 109 22341-50

[17] Qi Q, Liu H, Feng W, Tian H, Xu H and Huang X 2015 Theoretical investigation on the interaction of subnano platinum clusters with graphene using DFT methods Computational Materials Science 96 268-76

[18] Rajesh C and Majumder C 2019 Interaction of gold clusters with graphene and graphene layer over Ni(111) surface: A density functional study Applied Surface Science 469 917-22

[19] Chan K T, Neaton J B and Cohen M L 2008 First-principles study of metal adatom adsorption on graphene Physical Review B - Condensed Matter and Materials Physics 77 1-12

[20] Ishii a, Yamamoto M, Asano H and Fujiwara K 2008 DFT calculation for adatom adsorption on graphene sheet as a prototype of carbon nanotube functionalization Journal of Physics: Conference Series 100052087

[21] Cao C, Wu M, Jiang J and Cheng H P 2010 Transition metal adatom and dimer adsorbed on graphene: Induced magnetization and electronic structures Physical Review B - Condensed Matter and Materials Physics 81 1-9

[22] Johll H, Kang H C and Tok E S 2009 Density functional theory study of $\mathrm{Fe}$, Co, and Ni adatoms and dimers adsorbed on graphene Physical Review B - Condensed Matter and Materials Physics 79 1-18

[23] Cabria I, López M J and Alonso J A 2010 Theoretical study of the transition from planar to three-dimensional structures of palladium clusters supported on graphene Physical Review B Condensed Matter and Materials Physics 81 1-5

[24] Rêgo C R C, Tereshchuk P, Oliveira L N and Da Silva J L F 2017 Graphene-supported small transition-metal clusters: A density functional theory investigation within van der Waals corrections Physical Review B 95 16-8

[25] Ostad F Z, Ghazi M E, Javan M and Izadifard M 2019 DFT study of Ptn, Pdn, and Irn ( $\mathrm{n}=5$, 6) clusters adsorbed on graphene: Structural and electronic properties Physica B: Condensed Matter 575411678 
[26] Sanchez-Paisal Y, Sanchez-Portal D and Ayuela A 2009 Ab initio calculations of zirconium adsorption and diffusion on graphene Physical Review B - Condensed Matter and Materials Physics $801-10$

[27] Ambrusi R E, Orazi V, Marchetti J M and Pronsato M E 2020 Ni clusters embedded in multivacancy graphene substrates Journal of Physics and Chemistry of Solids 138109258

[28] Zhang P, Song Q, Zhuang J and Ning X J 2017 The effects of metal-cluster on electronic transport of graphene with vacancy studied by first-principles Superlattices and Microstructures 109 47-53

[29] Jadoon T, Carter-Fenk K, Siddique M B A, Herbert J M, Hussain R, Iqbal S, Iqbal J and Ayub K 2020 Silver clusters tune up electronic properties of graphene nanoflakes: A comprehensive theoretical study Journal of Molecular Liquids 297111902

[30] Amft M, Lebègue S, Eriksson O and Skorodumova N V. 2011 Adsorption of Cu, Ag, and Au atoms on graphene including van der Waals interactions Journal of Physics Condensed Matter 23

[31] Ziambaras E, Kleis J, Schröder E and Hyldgaard P 2007 Potassium intercalation in graphite: A van der Waals density-functional study Physical Review B 76 1-10

[32] Grimme S 2006 Semiempirical GGA-Type Density Functional Constructed with a LongRange Dispersion Correction Journal of Computational Chemistry 27 1787-99

[33] Medeiros P V C, Gueorguiev G K and Stafström S 2012 Benzene, coronene, and circumcoronene adsorbed on gold, and a gold cluster adsorbed on graphene: Structural and electronic properties Physical Review B - Condensed Matter and Materials Physics 85 1-7

[34] Li X, Lin S, Lin X, Xu Z, Wang P, Zhang S, Zhong H, Xu W, Wu Z and Fang W 2016 Graphene/h-BN/GaAs sandwich diode as solar cell and photodetector Optics Express 24134

[35] Kuang Y, Liu Y, Ma Y, Xu J, Yang X, Hong X and Feng J 2015 Modeling and Design of Graphene GaAs Junction Solar Cell Advances in Condensed Matter Physics 2015 1-7

[36] Brenny B J M, Yang Y, Brown A S, Polman A, Coenen T, Everitt H O, Losurdo M and Knight M W 2015 Gallium Plasmonics: Deep Subwavelength Spectroscopic Imaging of Single and Interacting Gallium Nanoparticles ACS Nano 9 2049-60

[37] Losurdo M, Yi C, Suvorova A, Rubanov S, Kim T H, Giangregorio M M, Jiao W, Bergmair I, Bruno G and Brown A S 2014 Demonstrating the capability of the high-performance plasmonic gallium-graphene couple ACS Nano 8 3031-41

[38] Al Balushi Z Y, Miyagi T, Lin Y C, Wang K, Calderin L, Bhimanapati G, Redwing J M and Robinson J A 2015 The impact of graphene properties on GaN and AlN nucleation Surface Science 634 81-8

[39] Mach J, Procházka P, Bartošík M, Nezval D, Piastek J, Hulva J, Švarc V, Konečný M, Kormoš $\mathrm{L}$ and Šikola T 2017 Electronic transport properties of graphene doped by gallium Nanotechnology 28

[40] Gecim G, Ozekmekci M and Fellah M F 2020 Ga and Ge-doped graphene structures: A DFT study of sensor applications for methanol Computational and Theoretical Chemistry 1180 112828

[41] Blöchl P E 1994 Projector augmented-wave method Physical Review B 50 17953-79

[42] Kresse G 1999 From ultrasoft pseudopotentials to the projector augmented-wave method Physical Review B 59 1758-75

[43] Kresse G and Hafner J. 1993 Ab initio molecular dynamics for liquid metals Physical Review 


\section{B $47558-61$}

[44] Kresse G and Furthmüller J 1996 Efficient iterative schemes for ab initio total-energy calculations using a plane-wave basis set Physical Review B 54 11169-86

[45] Kresse G and Furthmuller J 1996 Efficiency of ab-initio total energy calculations for metals and semiconductors using a plane-wave basis set av * Computational Materials Science 6 1550

[46] Perdew J P, Burke K, Ernzerhof M, of Physics D and Quantum Theory Group Tulane University N O L 70118 J 1996 Generalized Gradient Approximation Made Simple Physical Review Letters 77 3865-8

[47] Monkhorst H J and Pack J D 1976 Special points for Brillouin-zone integrations Physical Review B 13 5188-92

[48] Jalkanen J P, Halonen M, Fernández-Torre D, Laasonen K and Halonen L 2007 A computational study of the adsorption of small $\mathrm{Ag}$ and Au nanoclusters on graphite Journal of Physical Chemistry A 111 12317-26

[49] Sk E [Slides] Bader Analysis : Calculating the Charge on Individual Atoms in Molecules \& Crystals Molecules 0-24

[50] Lehtinen P O, Foster A S, Ma Y, Krasheninnikov A V. and Nieminen R M 2004 IrradiationInduced Magnetism in Graphite: A Density Functional Study Physical Review Letters 93 1-4

[51] Geim a K and Novoselov K S 2007 The rise of graphene. Nature materials 6 183-91 